\title{
Caracterización de Alteraciones Ultrasonográficas Prostáticas en Perros Atendidos en una Clínica Veterinaria en Lima, Perú, entre 2010 y 2014
}

\author{
Characterization of Prostatic Ultrasonographic Abnormalities in Dogs \\ Treated in a Veterinary Clinic in Lima, Peru from 2010 to 2014
}

\author{
Gabriela Ygreda $^{1,2}$, Ricardo Grandez ${ }^{1.2}$, Roberto Valencia ${ }^{1}$
}

\section{Resumen}

El presente estudio caracteriza las alteraciones ultrasonográficas prostáticas en perros atendidos en la Clínica Veterinaria Docente Cayetano Heredia (Lima, Perú) entre 2010 y 2014, correlacionando las alteraciones observadas con los diagnósticos compatibles a patologías prostáticas, considerando las variables tamaño, edad y raza. Se utilizaron 407 historias clínicas de pacientes que presentaron una o más alteraciones prostáticas, siendo la población más afectada los perros adultos, de tamaño pequeño y raza pura; asimismo, la condición más común fue la prostatomegalia. La hiperplasia prostática benigna y las neoplasias prostáticas fueron las alteraciones anatomopatológicas que más predominaron. Las alteraciones prostáticas también pudieron ser evidenciadas en animales jóvenes. Los resultados reafirman que la ultrasonografía permite la identificación de las patologías prostáticas más frecuentes.

Palabras clave: ultrasonografía, próstata, canes

\section{Abstract}

The aim of this study was to characterize the prostatic ultrasonographic alterations in dogs treated at the Veterinary Clinic Cayetano Heredia (Lima, Peru), during 2010 to 2014, correlating the observed alterations with diagnoses compatible to prostatic pathologies, considering the size, age and breed. A total of 407 clinical histories of patients that presented one or more prostatic alterations were evaluated, being the adult dogs of small size and purebred the most affected population, and the prostatomegaly was the most common condition. Benign prostatic hyperplasia and prostatic neoplasias were the

\footnotetext{
${ }^{1}$ Facultad de Medicina Veterinaria y Zootecnia, Universidad Peruana Cayetano Heredia, Lima, Perú

${ }^{2}$ E-mail: gabriela.ygreda@upch.pe
}

Recibido: 20 de setiembre de 2016

Aceptado para publicación: 12 de enero de 2017 
predominant anatomopathologic alterations. Prostatic alterations can also be seen in young animals. The results reaffirm that the ultrasonography allows the identification of the most frequent prostatic pathologies.

Key words: ultrasonography, prostate, dog

\section{INTRODUCCIÓN}

La ultrasonografía es una técnica simple de exploración rápida, no invasiva, mediante la cual se puede observar la arquitectura de órganos explorables, simetría, posición y forma; además de ayudar en la toma de muestras mediante la aspiración dirigida o biopsia de lesiones encontradas (Fritsch y Gerwing, 1996; Nyland, 2004).

La próstata es una glándula sexual accesoria del perro macho, que produce y almacena la mayor parte del líquido del eyaculado (Mir y Fontbonne, 2010). Dicha función secretoria aumenta hasta los cuatro años de edad, donde comienza a declinar (Verstegen, 1998).

Las patologías de la próstata son comunes y suelen tener origen infeccioso y hormonal, así como por alteraciones anatómicas y embriológicas. Las enfermedades prostáticas más frecuentes son la hiperplasia prostática benigna, la prostatitis bacteriana, los quistes prostáticos, los abscesos prostáticos y las neoplasias prostáticas (Krawiec y Heflin, 1992; Avedaño, 2008; Smith, 2008). Se ha observado que la incidencia de patologías prostáticas se incrementa con la edad del animal, lo cual podría estar relacionado con el mayor desarrollo fisiológico del órgano bajo la influencia de los andrógenos (Root-Kustitz y Klausner, 2000).

El presente estudio determinó la prevalencia de enfermedades prostáticas que se presentaron en perros atendidos en la Clínica Veterinaria Docente Cayetano Heredia, entre 2010 y 2014.

\section{Materiales y Métodos}

Se trabajó con las historias clínicas e informes ecográficos de archivo digital de perros atendidos en el periodo 2010-2014, en la Clínica Veterinaria Docente Cayetano Heredia (CVDCH), de la Facultad de Medicina Veterinaria y Zootecnia, Universidad Peruana Cayetano Heredia, ubicada en el distrito de San Martín de Porres, Lima, Perú. Los procedimientos ecográficos fueron realizados con un equipo Esaote, Pye Medical, modelo 100 Falco Vet con transductor multifrecuencia de 5-7.5 MHz hasta enero de 2011 y con un ecógrafo Sonosite, Micromaxx, transductor sectorial 4-8 MHz a partir de febrero de 2011.

Los registros incluidos en el estudio comprendieron los casos propios de la CVDCH $\mathrm{y}$ de los pacientes derivados a diagnóstico ecográfico por solicitud del médico veterinario tratante. Las ecografías fueron realizadas por el personal médico veterinario (tres ecografistas). Los informes utilizados cumplieron los siguientes criterios: tener los datos completos de la reseña, al menos una ecografía abdominal, y corresponder a perros machos no castrados con evaluación prostática y con medición del riñón izquierdo.

De 1289 historias clínicas, 563 cumplieron con los criterios de inclusión. Los datos de los pacientes y sus propietarios se mantuvieron en el anonimato. Los informes fueron clasificados de acuerdo a las variables edad $(<1.5, \geq 1.5$ y $<6, \geq 6$ y $<12, \geq 12$ años), raza (pura, cruce) y tamaño según el peso (pequeño [ $<8 \mathrm{~kg}$ ], mediano [ $8 \mathrm{y}<18 \mathrm{~kg}$ ], grande [>18 y $40 \mathrm{~kg}])$. Asimismo, se consideraron las variables ubicación, tamaño, forma, 
ecogenicidad, ecotextura y presencia de áreas cavitarias.

El criterio para el tamaño normal de la próstata estuvo de acuerdo al tamaño del perro. Así, $2.14 \pm 0.46 \mathrm{~cm}$ para canes pequeños, $2.90 \pm 0.98 \mathrm{~cm}$ para canes medianos, y $3.44 \pm 0.72 \mathrm{~cm}$ para canes grandes (Miguel del Priego, 2007).

La ecotextura fue definida como conservada, aumentada, disminuida o heterogénea; la ecogenicidad fue clasificada en conservada, homogénea e hipoecogénica, homogénea e hiperecogénica, o heterogénea. La forma prostática se clasificó en conservada o anormal, y la ubicación se definió como conservada (pélvica), abdominal o perineal. La cantidad de áreas cavitarias fueron identificadas como estructuras regulares o irregulares de contenido principalmente anaecoico, determinándose si eran únicas o múltiples y el tamaño relativo de las mismas.

Los casos clínicos fueron clasificados de acuerdo a los criterios descritos, permitiendo clasificar a los pacientes en sanos o enfermos. En los perros clasificados como enfermos se determinó una compatibilidad diagnóstica de acuerdo las alteraciones anatomopatológicas predominantes: hiperplasia prostática benigna, neoplasia prostática, prostatitis con quistes o abscesos, hiperplasia prostática benigna con quistes o abscesos, prostatitis, atrofia prostática, hiperplasia prostática benigna con quistes o abscesos paraprostáticos, quistes, prostatitis con quistes o abscesos paraprostáticos, y cálculo prostático (Avedaño, 2008; Smith, 2008). Posteriormente, se evaluó la frecuencia de las alteraciones ecográficas prostáticas, según el tamaño, edad y raza de los canes.

\section{Resultados}

De las 563 historias clínicas que cumplieron con los criterios de inclusión, 407 fue- ron de perros con una o más alteraciones prostáticas. La mayoría de estas alteraciones se presentaron en perros entre 6 y 12 años $(62.7 \%)$, de tamaño pequeño (48.9\%), y de raza pura (63.1\%) (Cuadro 1).

En cuanto a las características ultrasonográficas, se observó que predominaron las alteraciones en el tamaño prostático $(90.2 \%)$ y de ecogenicidad (68.1\%), siendo de menor frecuencia las alteraciones de la arquitectura prostática (47.7\%) (Cuadro 1). La prostatomegalia fue la condición de tamaño más común $(83.8 \%)$, mientras que la atrofia prostática en general se presentó mayormente en perros de 12 años a más (23.1\%).

En el análisis de compatibilidad de las alteraciones ultrasonográficas con patologías específicas (Cuadros 2 y 3 ), predominaron la presentación de alteraciones compatibles con hiperplasia prostática benigna $(28.7 \%$; Figura $1 \mathrm{~A})$, con neoplasias (16.7\%; Figura 1B) y con prostatitis con quistes o abscesos $(16.5 \%$; Figura 2).

Para la variable edad, los perros menores de 1.5 años presentaron mayor frecuencia de prostatitis con quistes o abscesos (36.2\%). Los perros entre 1.5 y 6 años, y entre 6 a 12 años presentaron en su mayoría hiperplasia prostática benigna (32.6 y 29.4\%, respectivamente). Asimismo, los perros de tamaño pequeño presentaron en su mayoría hiperplasia prostática benigna (41.2\%), los de tamaño mediano presentaron mayormente lesiones asociadas a neoplasias prostáticas (22.3\%), y los perros grandes presentaron prostatitis con quistes o abscesos (24.4\%). Por otro lado, los perros de raza pura y cruzados presentaron mayormente hiperplasia prostática benigna (Cuadro 2). Finalmente, las principales alteraciones de ubicación fueron el desplazamiento de la próstata a la región perineal $(2.9 \%)$ y a la cavidad abdominal $(0.7 \%)$. 
Cuadro 1. Frecuencia relativa de presentación de alteraciones de las características ultrasonográficas prostáticas en 407 perros evaluados en la Clínica Veterinaria Docente Cayetano Heredia, Facultad de Medicina Veterinaria y Zootecnia, Universidad Peruana Cayetano Heredia, durante los años 2010 y 2014 (Lima, Perú)

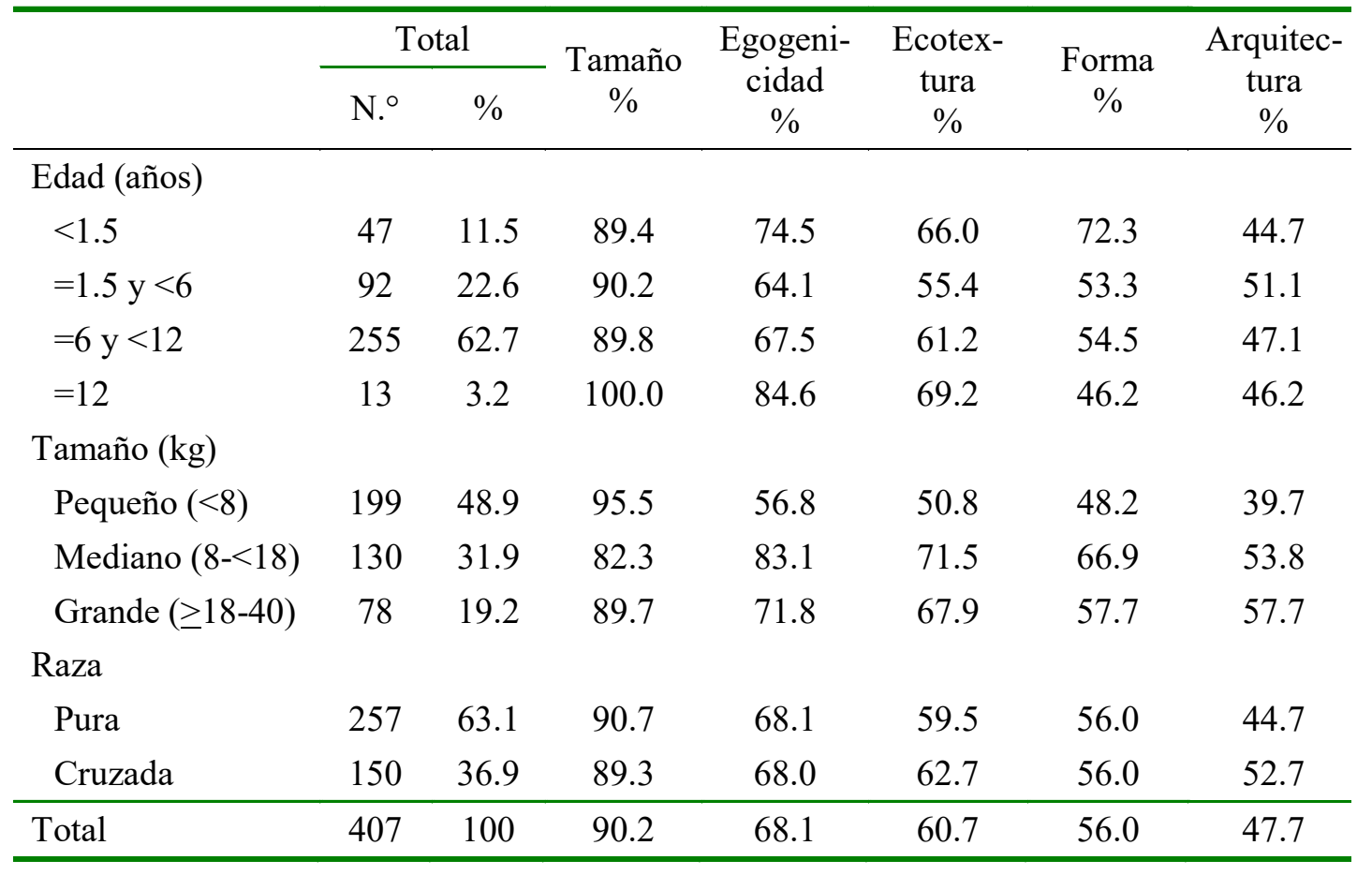

\section{Discusión}

La mayor frecuencia de casos con alteraciones de próstata en el presente trabajo en relación al estudio de Avedaño (2008), se podría deber a que los pacientes considerados en este estudio acudieron al servicio de ecografía referenciados por su médico veterinario tratante o por sospecha de una patología abdominal o pélvica, a diferencia del otro estudio, donde la población correspondió a una campaña de salud, siendo animales no necesariamente enfermos. Por otro lado, en el estudio de Peña et al. (2013) se observó una mayor frecuencia de animales con patologías prostáticas, ya que incluyó ultrasonografía y citología complementaria, permitiendo el diagnóstico de un mayor número de patologías, debido a la comple- mentación con la técnica citológica que identificó patologías que no necesariamente originaron alteraciones a la ultrasonografía.

En el presente estudio, la población adulta y de tamaño pequeño fue la más frecuente, debido a que, en la práctica actual, la mayoría de perros llevados a consulta corresponde a edades entre 6 y 12 años (Goyache, 2012), así como a la tendencia de tener perros de razas pequeñas por su facilidad de cuidado (Escalante, 2012).

Las alteraciones de tamaño prostático son comunes en caninos a partir de los 5 años de edad, afectando al 70\% de perros (García, 2013), además que la mayoría de enfermedades prostáticas se caracteriza por desarrollar prostatomegalia (Lattimer, 1994; Barsanti 
Cuadro 2. Frecuencia absoluta y relativa de presentación de compatibilidad de alteraciones anatomopatológicas con patologías prostáticas: hiperplasia prostática benigna y prostatitis de perros con alteraciones prostáticas evaluados en la Clínica Veterinaria Docente Cayetano Heredia ( $\mathrm{n}=407)$. Lima, Perú

\begin{tabular}{|c|c|c|c|c|c|c|c|c|c|c|c|c|c|c|}
\hline & \multicolumn{2}{|c|}{ Total } & \multicolumn{2}{|c|}{ НРB } & \multicolumn{2}{|c|}{$\mathrm{HPB} \mathrm{Q} / \mathrm{A}$} & \multicolumn{2}{|c|}{$\begin{array}{l}\text { HPB } \\
\text { Q/A P }\end{array}$} & \multicolumn{2}{|c|}{ PTS } & \multicolumn{2}{|c|}{ PTS Q/A } & \multicolumn{2}{|c|}{$\begin{array}{c}\text { PTS } \\
\text { Q/A P }\end{array}$} \\
\hline & $\mathrm{n}$ & $\%$ & $\mathrm{n}$ & $\%$ & $\mathrm{n}$ & $\%$ & $\mathrm{n}$ & $\%$ & $\mathrm{n}$ & $\%$ & $\mathrm{n}$ & $\%$ & $\mathrm{n}$ & $\%$ \\
\hline \multicolumn{15}{|l|}{ Edad (años) } \\
\hline$<1.5$ & 47 & 12 & 10 & 21.3 & 3 & 6.4 & 1 & 2.1 & 11 & 23.4 & 17 & 36.2 & 0 & 0 \\
\hline$\geq 1.5-<6$ & 92 & 23 & 30 & 32.6 & 14 & 15.2 & 4 & 4.3 & 8 & 8.7 & 14 & 15.2 & 0 & 0 \\
\hline$\geq 6-<12$ & 255 & 63 & 75 & 29.4 & 35 & 13.7 & 3 & 1.2 & 33 & 12.9 & 34 & 13.3 & 4 & 1.6 \\
\hline$\geq 12$ & 13 & 3.2 & 2 & 15.4 & 3 & 23.1 & 0 & 0 & 1 & 7.7 & 2 & 15.4 & 0 & 0 \\
\hline
\end{tabular}

Tamaño

$(\mathrm{kg})^{1}$

\begin{tabular}{lcccccccccccccc} 
Pequeño & 199 & 49 & 82 & 41.2 & 25 & 12.6 & 2 & 1.0 & 20 & 10.1 & 26 & 13.1 & 1 & 0.5 \\
Mediano & 130 & 32 & 20 & 15.4 & 19 & 14.6 & 3 & 2.3 & 22 & 16.9 & 22 & 16.9 & 0 & 0 \\
$\quad$ Grande & 78 & 19 & 15 & 19.2 & 11 & 14.1 & 3 & 3.8 & 11 & 14.1 & 19 & 24.4 & 3 & 3.8 \\
Raza & & & & & & & & & & & & & & \\
$\quad$ Pura & 257 & 63 & 74 & 28.8 & 31 & 12.1 & 6 & 2.3 & 38 & 14.8 & 41 & 16.0 & 1 & 0.4 \\
Cruzado & 150 & 37 & 43 & 28.7 & 24 & 16.0 & 2 & 1.3 & 15 & 10.0 & 26 & 17.3 & 3 & 2.0 \\
\hline Total & 407 & 100 & 117 & 28.7 & 55 & 13.5 & 8 & 2.0 & 53 & 26.6 & 67 & 16.5 & 4 & 1.0 \\
\hline
\end{tabular}

HPB: Hiperplasia prostática benigna; HPB Q/A: Hiperplasia prostática benigna con quistes o abscesos; HPB Q/A P: Hiperplasia prostática benigna con quistes o abscesos paraprostáticos; PTS: Prostatitis; PTS Q/A: Prostatitis con quistes o abscesos; PTS Q/A P: Prostatitis con quistes o abscesos paraprostáticos

1 Pequeño [<8 kg], mediano [8 y <18 kg], grande [ $\geq 18$ y $40 \mathrm{~kg}$ ]

y Finco, 1995). El agrandamiento prostático se puede deber a diversas condiciones patológicas como la prostatitis, abscesos prostáticos, hiperplasia prostática benigna, quistes prostáticos y neoplasia prostática (LeRoy y Northrup, 2009); hallazgos diagnósticos que fueron comunes en el presente estudio.

Teske et al. (2002) encontró que el $57.1 \%$ de perros presentaba hiperplasia prostática benigna confirmada por citología, siendo esta la anormalidad prostática más común; además, reportaron que el 13\% de las patologías prostáticas correspondían a neoplasias, lo cual coincidió con los resultados del presente estudio.
La presentación de lesiones compatibles con prostatitis con quistes o abscesos fue de $36.2 \%$ del total de prevalencia, porcentaje similar al reportado por Black et al. (1998), donde el $42.0 \%$ de las próstatas con quistes tenían evidencia de infecciones bacterianas. Algunos autores mencionan que la hiperplasia prostática benigna se inicia como una hiperplasia glandular a los 3 años de edad (Kutzler y Yeager, 2005), lo cual explicaría los hallazgos del presente estudio donde los perros jóvenes tienen un mayor porcentaje para esta patología.

Diversos estudios demuestran que los animales de razas medianas o grandes son los más afectados con las patologías 
Cuadro 3. Frecuencia absoluta y relativa de presentación de compatibilidad de alteraciones anatomopatológicas con patologías prostáticas: neoplasias, atrofia prostática, quistes y cálculos prostaticos en perros con alteraciones prostáticas, evaluados en la Clínica Veterinaria Docente Cayetano Heredia $(n=407)$. Lima, Perú

\begin{tabular}{|c|c|c|c|c|c|c|c|c|c|c|c|}
\hline & & \multicolumn{2}{|c|}{ Total } & \multicolumn{2}{|c|}{ NEO } & \multicolumn{2}{|c|}{ ATRO } & \multicolumn{2}{|c|}{ QUIS } & \multicolumn{2}{|c|}{ CAL } \\
\hline & & $\mathrm{n}$ & $\%$ & $\mathrm{n}$ & $\%$ & $\mathrm{n}$ & $\%$ & $\mathrm{n}$ & $\%$ & $\mathrm{n}$ & $\%$ \\
\hline \multicolumn{12}{|c|}{ Edad (años) } \\
\hline & $<1.5$ & 47 & 12 & 0 & 0.0 & 5 & 10.6 & 0 & 0.0 & 0 & 0.0 \\
\hline & $\geq 1.5-<6$ & 92 & 23 & 16 & 17.4 & 5 & 5.4 & 1 & 1.1 & 0 & 0.0 \\
\hline & $\geq 6-<12$ & 255 & 63 & 50 & 19.6 & 13 & 5.1 & 6 & 2.4 & 2 & 0.8 \\
\hline & $\geq 12$ & 13 & 3.2 & 2 & 15.4 & 3 & 23.1 & 0 & 0.0 & 0 & 0.0 \\
\hline \multicolumn{12}{|c|}{ Tamaño $(\mathrm{kg})$} \\
\hline & $\begin{array}{l}\text { Pequeño } \\
(<8)\end{array}$ & 199 & 49 & 29 & 14.6 & 10 & 5.0 & 4 & 2.0 & 0 & 0.0 \\
\hline & $\begin{array}{l}\text { Mediano } \\
(8-<18)\end{array}$ & 130 & 32 & 29 & 22.3 & 11 & 8.5 & 2 & 1.5 & 2 & 1.5 \\
\hline & $\begin{array}{l}\text { Grande } \\
(\geq 18-40)\end{array}$ & 78 & 19 & 10 & 12.8 & 5 & 6.4 & 1 & 1.3 & 0 & 0.0 \\
\hline \multicolumn{12}{|l|}{ Raza } \\
\hline & Pura & 257 & 63 & 43 & 16.7 & 17 & 6.6 & 4 & 1.6 & 2 & 0.8 \\
\hline & Cruzado & 150 & 37 & 25 & 16.7 & 9 & 6.0 & 3 & 2.0 & 0 & 0.0 \\
\hline Total & & 407 & 100 & 68 & 16.7 & 26 & 6.4 & 7 & 1.7 & 2 & 0.5 \\
\hline
\end{tabular}

NEO: Neoplasia prostática; ATRO: Atrofia prostática; QUIS: Quistes; CAL: Cálculo prostático

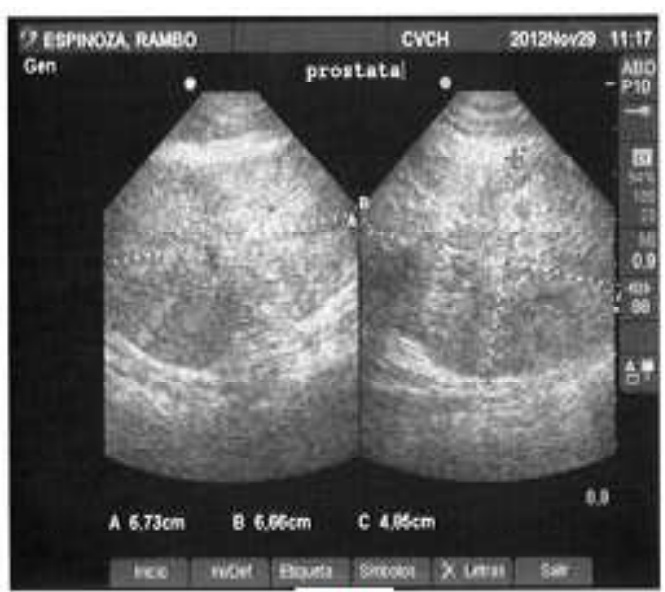

(A)

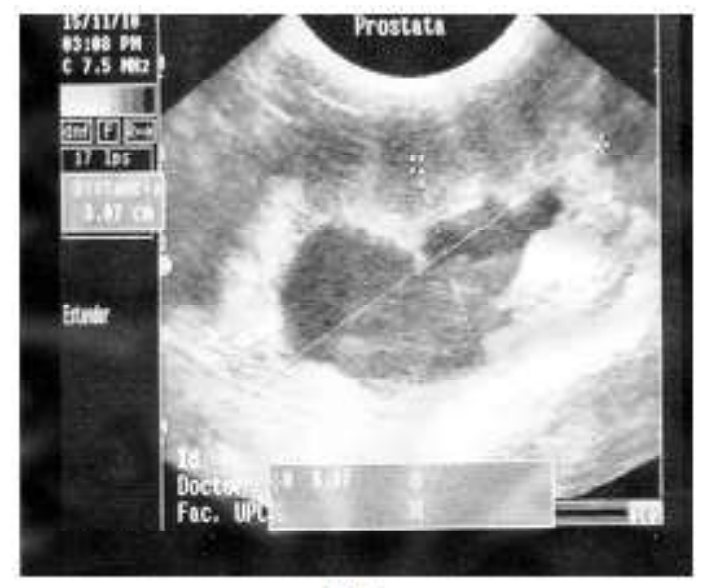

(B)

Figura 1.Registro fotográfico de próstata canina. (A) Corte transversal (izquierda) y longitudinal (derecha) evidenciando prostatomegalia, ecotextura de granularidad aumentada y ecogenicidad levemente heterogénea. Compatible con hiperplasia prostática benigna. (B) Corte longitudinal evidenciando prostatomegalia con patrón complejo invadiendo parcialmente cuello y lumen vesical. Compatible con neoplasia prostática 


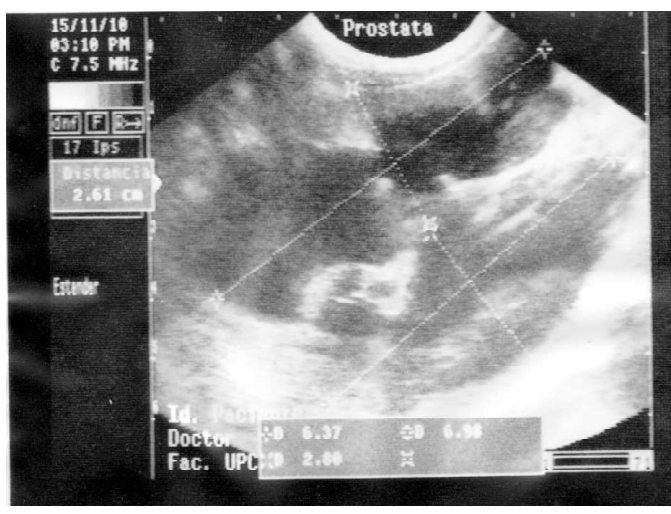

Figura 2. Registro fotográfico de próstata canina en corte longitudinal evidenciando prostatomegalia, ecogenicidad y ecotextura marcadamente heterogénea y presencia de áreas cavitarias coalescentes. Compatible con quistes o abscesos prostáticos

prostáticas en general, especialmente el Doberman (Ferreira, 2011) y el Pastor Alemán (Johnston et al., 2001); sin embargo, en el presente estudio fueron los perros pequeños los que presentaron mayor frecuencia, lo cual podría deberse a la mayor población de animales de este tamaño y a su longevidad.

\section{Conclusiones}

- La población más afectada por alteraciones prostáticas correspondió a perros adultos, de tamaño pequeño y de raza pura, siendo la prostatomegalia la condición más común.

- Las alteraciones anatomopatológicas predominantes fueron las compatibles con hiperplasia prostática benigna y neoplasias prostáticas.

\section{Literatura Citada}

1. Avedaño A. 2008. Evidencia de alteraciones anatomopatológicas prostáticas detectadas por ultrasonografía transab- dominal en canes adultos enteros aparentemente sanos. Tesis de Médico Veterinario Zootecnista. Lima: Universidad Peruana Cayetano Heredia. $29 \mathrm{p}$.

2. Barsanti JA, Finco DR. 1995. Prostatic diseases. In: Ettinger SJ, Feldman EC (eds). Textbook of veterinary internal medicine: diseases of the dog and cat. $4^{\text {th }}$ ed. Philadelphia, USA: WB Saunders. p 1662-1685.

3. Black GM, Ling GV, Nyland TG, Baker T. 1998. Prevalence of prostatic cysts in adult, large-breed dogs. J Am Anim Hosp Assoc 34: 177-180. doi: 10.5326/15473317-34-2-177

4. Escalante C. 2012. Evaluación de la compra de perros con pedigrí de razas pequeñas para la creación de una empresa reproductora y comercializadora en la ciudad de Guayaquil. Tesis de Licenciatura en Marketing y Publicidad. Guayaquil: Univ de Guayaquil. 112 p.

5. Ferreira D. 2011. Affecoes prostáticas em caes. Tesis de Médico Veterinario. Porto Alegre, Brasil: Univ Federal do Rio Grande do Sul. 50 p.

6. Fritsch R, Gerwing M. 1996. Ecografía de perros y Gatos. España: Acribia. 246 p.

7. García F. 2013. Cirugía de la próstata canina. Selecciones Veterinarias. [Internet]. Disponible en: http:// seleccionesveterinarias.intermedica.com.ar/ es/articulos/reproduccion-animal/cirugiade-la-prostata-canina

8. Goyache J. 2012. Revisiones del estado de salud en animales de compañía. Colegio Oficial de Veterinarios de Madrid. [Internet]. Disponible en http://www.colvema.org/WV_descargas/goyacheok-07042012162529.pdf

9. Johnston SD, Root Kustriz MV, Olson $P N$. 2001. Disorders of the canine prostate. In: Canine and feline theriogenology. Philadelphia, USA: WB Saunders. p 337-355.

10. Krawiec DR, Heflin D.1992. Study of prostatic disease in dogs: 177 cases (1981-1986). J Am Vet Med Assoc 15: 1119-1122. 
11. Kutzler M, Yeager A. 2005. Prostatic diseases. In: Textbook of veterinary internal medicine. $6^{\text {th }}$ ed. St. Louis, Missouri, USA: WB Saunders. p 18091819.

12. Lattimer JC. 1994. The prostate gland. In: Thrall DE (ed). Textbook of veterinary diagnostic radiology. $2^{\text {nd }} e d$. Philadelphia, USA: WB Saunders. p 479-493.

13. LeRoy B, Northrup N. 2009. Prostate cancer in dogs: comparative and clinical aspects. Vet J 180: 149-162. doi: 10.1016/ j.tvj1.2008.07.012

14. Miguel de Priego C. 2007. Correlación entre el tamaño ecográfico de la glándula prostática y el riñón izquierdo en caninos. Tesis de Médico Veterinario Zootecnista. Lima. Univ Peruana Cayetano Heredia. 16 p.

15. Mir F, Fontbonne A. 2010. Retorno de la fertilidad en un perro con un absceso prostático. Clin Vet Peq Anim 30: 109-113.

16. Nyland TG 2004. Próstata y testículos. En: Nyland TG, Matoon JS (eds). Diagnóstico ecográfico en pequeños animales. $2^{a}$ ed. España: Multimédica Ediciones Veterinarias. p 20-276.

17. Peña S, Chavera A, Cahua J, Perales R. 2013. Alteraciones prostáticas en caninos determinadas mediante ultrasono- grafía y citología por aspiración eco-guiada. Rev Inv Vet Perú 24: 207-216. doi: 10.15381/rivep.v24i2.2502

18. Root-Kustitz M, Klausner J. 2000. Prostatic disease. In: Ettinger S, Feldman E (eds). Textbook of veterinary internal medicine. Philadelphia, USA: WB Saunders. p 1687-1696.

19. Ruel Y, Barthez PY, Mailles A, Begon D. 1998. Ultrasonographic evaluation of the prostate in healthy intact dogs. Vet Radiol Ultrasound 39: 212-216. doi: 10.1111/j.1740-8261.1998.tb00342.x

20. Smith J. 2008. Canine prostatic disease: A review of anatomy, pathology, diagnosis, and treatment. Theriogenology 70 : 375-83. doi: 10.1016/j theriogenology.2008.04.039

21. Teske E, Naan EC, van Dijk EM, van Garderen E, Schalken JA. 2002. Canine prostate carcinoma: epidemiological evidence of an increased risk in castrated dogs. Moll Cell Endocrinol 197: 251-255. doi: 10.1016/S0303-7207(02)00261-7

22. Verstegen J. 1998. Conditions of the male. In: Manual of small animals reproduction and neonatology. Simpson G, England G, Harvey M (eds). London: British Small Animal Association. p 71-83. 